\title{
New sheath nematode, Hemicycliophora paraconida n. sp. (Rhabditida: Hemicycliophoridae) from Northern forest in Iran
}

\author{
ESMAEIL Miraeiz, ${ }^{1}$ HADi GHORbanZAD, ${ }^{2}$ MEHRAB ESMAEILI, ${ }^{3}$ \\ RAMIN HEYDARI ${ }^{4}$
}

\footnotetext{
${ }^{1}$ Department of Plant Protection, College of Agriculture and Natural Resources, University of Tehran, Karaj, Iran, ORCID: 00000001-5926-3196

2 Department of Plant Protection, College of Agriculture and Natural Resources, University of Tehran, Karaj, Iran, ORCID: 00000003-3506-4162

${ }^{3}$ Department of Plant Protection, College of Agriculture and Natural Resources, University of Tehran, Karaj, Iran, ORCID: 00000002-5890-0242

${ }^{4}$ Department of Plant Protection, College of Agriculture and Natural Resources, University of Tehran, Karaj, Iran, ORCID: 00000002-9847-089X
}

Corresponding author e-mail: rheydari@ut.ac.ir

Keywords 28S D2/D3, Hemicycliophoroidea, morphology, morphometric, new species, taxonomy

Abstract Hemicycliophora paraconida n. sp. from the Tilekenar forest in northern Iran, is described and illustrated. The new species is characterized by having female body length of 907-1197 $\mu \mathrm{m}$, 286-341 body annuli and a 96-115 $\mu \mathrm{m}$ long stylet. Lateral fields with three longitudinal lines forming two rows of blocks with breaks and anastomosis of transverse striae; each annulus in lateral field with four subcuticular ovate markings. Tail elongated uniformly conoid, with distinct annuli and narrowly rounded to acute terminus. Male possess robust and semi-circular spicules and a well-developed penial tube. Based on molecular and morphological comparisons, the new species comes close to seven species of the genus namely: $H$. conida, H. iwia, $H$. ovata, $H$. hellenica, $H$. poranga, $H$. ornamenta and $H$. halophila, but it differs from them by the body size, R related indices, stylet length, lateral field structure, vulva position and tail shape. Molecular analyses based on sequences of D2-D3 expansion segments of 28S rRNA gene supported its morphological status as a new species and revealed that the new species is closest to the $H$. conida clade in the dendrogram inferred using 28S D2-D3 gene.

Nowy gatunek nicienia, Hemicycliophora paraconida n. sp. (Rhabditida: Hemicycliophoridae) z lasu w Północnym Iranie

Słowa kluczowe 28S D2/D3, Hemicycliophoroidea, morfologia, morfometria, nowy gatunek, taksonomia

Streszczenie Wykonano opis wraz z ilustracjami nowego gatunku, Hemicycliophora paraconida n. sp. z lasu Tilekenar w północnym Iranie. Samica nowego gatunku charakteryzuje się długością ciała 907-1197 $\mu \mathrm{m}$, segmentami pierścieniowymi w liczbie 286-341 oraz sztyletem o długości 96-115 $\mu \mathrm{m}$. Pola boczne z trzema liniami podłużnymi tworzącymi dwa rzędy brodawek z przerwami i poprzecznych prążkami; każdy pierścień z boku ma cztery subkutikularne, 
owalne znamiona. Ogon wydłużony, jednolicie stożkowaty, z wyraźnymi pierścieniami i wąsko zwężający się do samego końca. Samce z mocnymi, półokrągłymi spikulami mają oraz dobrze rozwiniętym trzonem penisa. Na podstawie porównań molekularnych i morfologicznych nowy gatunek jest spokrewniony z siedmioma gatunkami z tego samego rodzaju: $H$. conida, H. iwia, H. ovata, H. hellenica, H. poranga, H. ornamenta i H. halophila, od których różni się wielkością ciała, wskaźnikami R, długością sztyletu, strukturą pola bocznego, usytuowaniem otworu genitalnego i kształtem ogona. Analizy molekularne oparte na sekwencjach segmentów D2-D3 genu 28S rRNA potwierdziły jego odmienność morfologiczną jako nowego gatunku i usytuowały go najbliżej kladu $H$. conida w dendrogramie skonstruowanym na podstawie genu 28S D2-D3.

\section{Introduction}

The plant-parasitic nematodes of the superfamily Hemicycliophoroidea Skarbilovich, 1959 commonly known as 'sheath nematodes. These nematodes are a group of obligate ectoparasitic nematodes. Many species belonging to the superfamily have been found associated with agricultural crops, although pathogenicity has only been studied for a few species (van den Berg et al., 2018). Some species of Hemicycliophora e.g., H. arenaria Raski, 1958, H. conida Thorne, 1955, H. parvana Tarjan, 1952, H. poranga Monteiro \& Lordello, 1978, H. similis Thorne 1955, H. typica de Man, 1921 have been reported as damaging agricultural crops such as carrot, celery, citrus, maple and tomato in several countries (Chitambar \& Subbotin, 2014). Host symptoms produced in response to nematode feeding are not well-known for most species within the genus. Some species could induce root galls, while the others, such as H. typica, may induce only slight swellings or no symptoms. Stubby root symptoms on carrots was induced by H. typica in sandy soil and H. conida caused host stunt and aberrant root development in The Netherlands (Chitambar, 1993).

Considering synonymy by Raski and Luc (1987), the superfamily includes two families with three genera i.e. Hemicycliophora de Man 1921, Caloosia Siddiqi \& Goodey, 1964 and Hemicaloosia Ray \& Das, 1978 (Chitambar, Subbotin, 2014). The taxonomic position of the genus Hemicycliophora has been changed by some authors since 1921 when de Man recovered a single male nematode specimen in a compost heap in Netherland and described it as a new species and genus. For a long time, males and females of this genus were been considered as two different nematodes by nematologists until Loos (1948) discovered males and females together in a population collected in Sri Lanka. The genus Hemicycliophora now belongs to subfamily Hemicycliophorinae and is known to have a global distribution with 135 valid species (Maria et al., 2018; Subbotin et al., 2014; van den Berg et al., 2018). The subfamily Hemicycliophorinae including the monotypic genus, is characterized by the presence of an extra cuticular layer adpressed or loose from the inner cuticle along the body. The lip region has two or three lip annuli which are not modified or separated. Transverse vulval slit bearing lips which are mostly modified. Males have smooth and offset labial region and semi-circular, U- or hook-shaped spicule (Chitambar, Subbotin, 2014; Raski, Luc, 1987).

Loof (1984) described six new and one known species of Hemicycliophora based on material collected by Sturhan from different regions in Iran during 1970-1974. More recently, five other members of the genus have been reported from Iran (Ghaderi et al., 2019; Ghaderi et al., 2018). In a nematological survey conducted in a forest of Mazandaran province in northern Iran, a population of an unknown Hemicycliophora species was recovered from soil samples collected from the rhizosphere of grasses. It is herein described as $H$. paraconida $\mathrm{n}$. sp. through morphological observation and molecular characterization by the partial 28S D2-D3 rRNA gene sequence. 


\section{Materials and Methods}

Sampling, extracting, mounting and drawing. Specimens of Hemicycliophora paraconida n. sp. were obtained from soil samples collected around roots of grasses in Tilekenar Forest, Mazandaran province. To obtain a cleaner suspension of nematodes, the tray method was used (Whitehead, Hemming, 1965). Nematodes were handpicked under a stereomicroscope, heat-killed by adding boiling 4\% formalin solution, transferred to anhydrous glycerin (De Grisse, 1969), mounted on permanent slides and examined under a Nikon E200 light microscope. Drawings and measurements were made using a drawing tube attached to the microscope. Photographs were taken using a camera (Dino-Lite digital microscope) attached to the same microscope.

DNA extraction, PCR and sequencing. Total DNA was extracted from single individual nematodes using worm lysis buffer containing proteinase K (Williams et al., 1992) and DNA extracts were stored at $-20^{\circ} \mathrm{C}$. The forward D2A (5'-ACAAGTACCGTGAGGGAAAGTTG-3') and reverse D3B (5'-TCGGAAGGAACCAGCTACTA-3') primers were used for amplification and sequencing of the fragment of the $28 \mathrm{~S}$ rRNA gene (Nunn, 1992). Fifty $\mu \mathrm{L}$ of PCR mixture contained 1× Taq DNA polymerase incubation buffer, $2.5 \mathrm{mM} \mathrm{MgCl} 2,0.2 \mathrm{mM}$ of each dNTP, $0.5 \mu \mathrm{M}$ of each primers and $2 \mu \mathrm{L}$ of DNA. The PCR amplification profile consisted of $5 \mathrm{~min}$ at $95^{\circ} \mathrm{C} ; 35$ cycles of $30 \mathrm{~s}$ at $94^{\circ} \mathrm{C}, 45 \mathrm{~s}$ at $55^{\circ} \mathrm{C}$ and $2 \mathrm{~min}$ at $72^{\circ} \mathrm{C}$, followed by a final step of $10 \mathrm{~min}$ at $72^{\circ} \mathrm{C}$. Two $\mu \mathrm{L}$ of the PCR product was run on a $1.2 \%$ TBE buffered agarose gel $(100 \mathrm{~V}, 40 \mathrm{~min})$. The band of the correct size was excised and purified using QIAquick gel Extraction Kit (Qiagen: www.qiagen.com) and sequenced directly for both strands using the amplification primers with an ABI 3730XL sequencer (Macrogen Corporation, South Korea).

DNA sequence alignment and phylogenetic inference. The obtained sequences of the partial 28S D2-D3 region of the nematode specimen were aligned using the online version of MAFFT version 7 (http://mafft.cbrc.jp/alignment/server/) (Katoh, Standley, 2013) with those of the other Hemicycliophora species and some related genera available in GenBank using the BLAST homology search program. After manually trimming the alignment, the Gblocks program (version 0.91b) using all three less stringent parameters, a server tool at the Castresana Lab (http://molevol. cmima.csic.es/castresana/Gblocks_server.html), was used to eliminate poorly aligned regions or divergent positions. The best-fitted model of DNA evolution was obtained using MrModeltest 2 (Nylander, 2004). Phylogenetic analyses was then performed using the Akaike-supported model, a general time reversible model, including among-site rate heterogeneity and estimates of invariant sites $(\mathrm{GTR}+\mathrm{G}+\mathrm{I})$ together with the base frequency, the proportion of invariable sites, and the gamma distribution shape parameters and substitution rates. The tree topology was confirmed using MrBayes v3.2.3 (Ronquist \& Huelsenbeck, 2003) with four chains (three heated and one cold). The number of generations for the total analysis was set to $2 \times 10^{6}$, with the chain sampled every 100 generations, and the burn-in value was $25 \%$. The Markov chain MonteCarlo (MCMC) method within a Bayesian framework was used to estimate the posterior probabilities of the phylogenetic trees using 50\% majority rule (Larget, Simon, 1999). A maximum likelihood (ML) tree was also reconstructed by using RaxmlGUI 1.3 (Silvestro, Michalak, 2012) software using the same alignment and nucleotide substitution model as in the Bayesian inference in 1000 bootstrap (BS) replicates. The consensus tree were selected to represent the phylogenetic relationships with branch length and support level and visualized using Dendroscope V.3.2.8 (Huson, Scornavacca, 2012) and redrawn in Adobe ${ }^{\circledR}$ Photoshop ${ }^{\circledR} 7.0$ ME. Paratylenchus hamatus and Gracilacus sp. (Accession numbers: KF242218 and KM061782) were used as the outgroup taxa. The Bayesian 
posterior probability (BPP) and ML BS values exceeding 0.50 and 50\%, respectively, are given on appropriate clades in the format of BPP/ML BS.

\section{Results}

\section{Hemicycliophora paraconida $\mathrm{n} . \mathrm{sp}$.}

Table 1. Morphometrics of Hemicycliophora paraconida $\mathrm{n}$. sp. All measurements are in $\mu \mathrm{m}$ and in the form: mean \pm s.d. (range).

\begin{tabular}{|l|c|c|c|}
\hline \multirow{2}{*}{ Characters } & \multicolumn{2}{|c|}{ Female } & Male \\
\cline { 2 - 4 } & holotype & paratypes & paratypes \\
\hline $\mathrm{n}$ & - & 12 & 10 \\
\hline $\mathrm{L}$ & 933 & $1017 \pm 100(907-1197)$ & $973 \pm 21(944-994)$ \\
\hline $\mathrm{L}$ & 786 & $879 \pm 96(766-1047)$ & $801 \pm 21(773-820)$ \\
\hline $\mathrm{b}$ & 21 & $21 \pm 1(19.5-22.5)$ & $37 \pm 3.3(33-41)$ \\
\hline $\mathrm{c}$ & 4.6 & $5.3 \pm 0.5(4.6-6.1)$ & $7.5 \pm 0.8(6.8-8.4)$ \\
\hline $\mathrm{c}^{\prime}$ & 6.3 & $7.4 \pm 0.7(6.3-8.3)$ & $5.6 \pm 0.2(5.5-6)$ \\
\hline V or T & 3.9 & $3.3 \pm 0.3(2.8-3.9)$ & $6.5 \pm 0.5(6-7)$ \\
\hline $\mathrm{V}^{\prime}$ & 83.6 & $83.0 \pm 0.6(82-84)$ & $15 \pm 3(12-17)$ \\
\hline Head height & 99 & $96 \pm 2(94-99)$ & - \\
\hline Head width & 10 & $9.5 \pm 0.8(8.5-10.5)$ & $8.3 \pm 0.5(8-9)$ \\
\hline Stylet & 20.5 & $21 \pm 0.8(20-22)$ & $12 \pm 0.0$ \\
\hline Conus & 101 & $104 \pm 6(96-115)$ & - \\
\hline Shaft & 81 & $82.5 \pm 4(78-91)$ & - \\
\hline $\mathrm{m}$ & 20 & $21 \pm 3.5(16-27)$ & - \\
\hline DGO from base of knobs & 80.2 & $80 \pm 2.5(75-84)$ & - \\
\hline Anterior end to centre of median bulb & 17 & $13 \pm 2.2(11-17)$ & - \\
\hline Nerve ring from anterior end & 137 & $134 \pm 6.5(123-143)$ & $94 \pm 13(87-114)$ \\
\hline Excretory pore from anterior end & 170 & $159 \pm 8(147-170)$ & $131 \pm 4.2(128-134)$ \\
\hline Pharynx length & 195 & $188 \pm 17(150-213)$ & $165 \pm 8(154-171)$ \\
\hline Head-vulva & 201 & $190 \pm 9(176-201)$ & $130 \pm 16(113-145)$ \\
\hline Max. body diam. & 780 & $844 \pm 84(757-1002)$ & - \\
\hline Anal/cloacal body diam. & 45 & $48.5 \pm 3(44-53)$ & $27 \pm 2.5(24-30)$ \\
\hline Vulva - anus & 38 & $43 \pm 4(37-49)$ & $27 \pm 2.5(24-30)$ \\
\hline Ovary or testis length & 43 & $45 \pm 4.5(36-53)$ & - \\
\hline Tail length & 398 & $359 \pm 80(259-507)$ & $145 \pm 27(114-163)$ \\
\hline Rs ${ }_{t}$ & 147 & $138 \pm 11(118-150)$ & $172 \pm 5.5(166-179)$ \\
\hline $\mathrm{R}_{\text {ex }}$ & 32 & $30 \pm 3(23-33)$ & - \\
\hline $\mathrm{R}_{\text {pha }}$ & 57 & $52 \pm 4(46-57)$ & - \\
\hline RV(ant) & 59 & $53 \pm 5(44-59)$ & - \\
\hline Ran & 268 & $244 \pm 16(230-272)$ & - \\
\hline Rvan & 287 & $259 \pm 18(243-290)$ & - \\
\hline $\mathrm{R}$ & 19 & $15 \pm 2.5(12-19)$ & - \\
\hline & 338 & $304 \pm 21(286-341)$ & \\
\hline & & & \\
\hline
\end{tabular}


New sheath nematode, Hemicycliophora paraconida n. sp. (Rhabditida: Hemicycliophoridae)...

\begin{tabular}{|l|c|c|c|}
\hline VL/VB & 4.3 & $3.8 \pm 0.3(3.3-3.4)$ & - \\
\hline Spicule (arc line) & - & - & $55.5 \pm 2(54-58)$ \\
\hline $\begin{array}{l}\text { Spicule width (straight distance between } \\
\text { extremities) }\end{array}$ & - & - & $28 \pm 0.6(27-29.5)$ \\
\hline Penial tube length & - & - & $17 \pm 2(15-19)$ \\
\hline Gubernaculum length & - & - & $12 \pm 0.5(11.5-13)$ \\
\hline
\end{tabular}

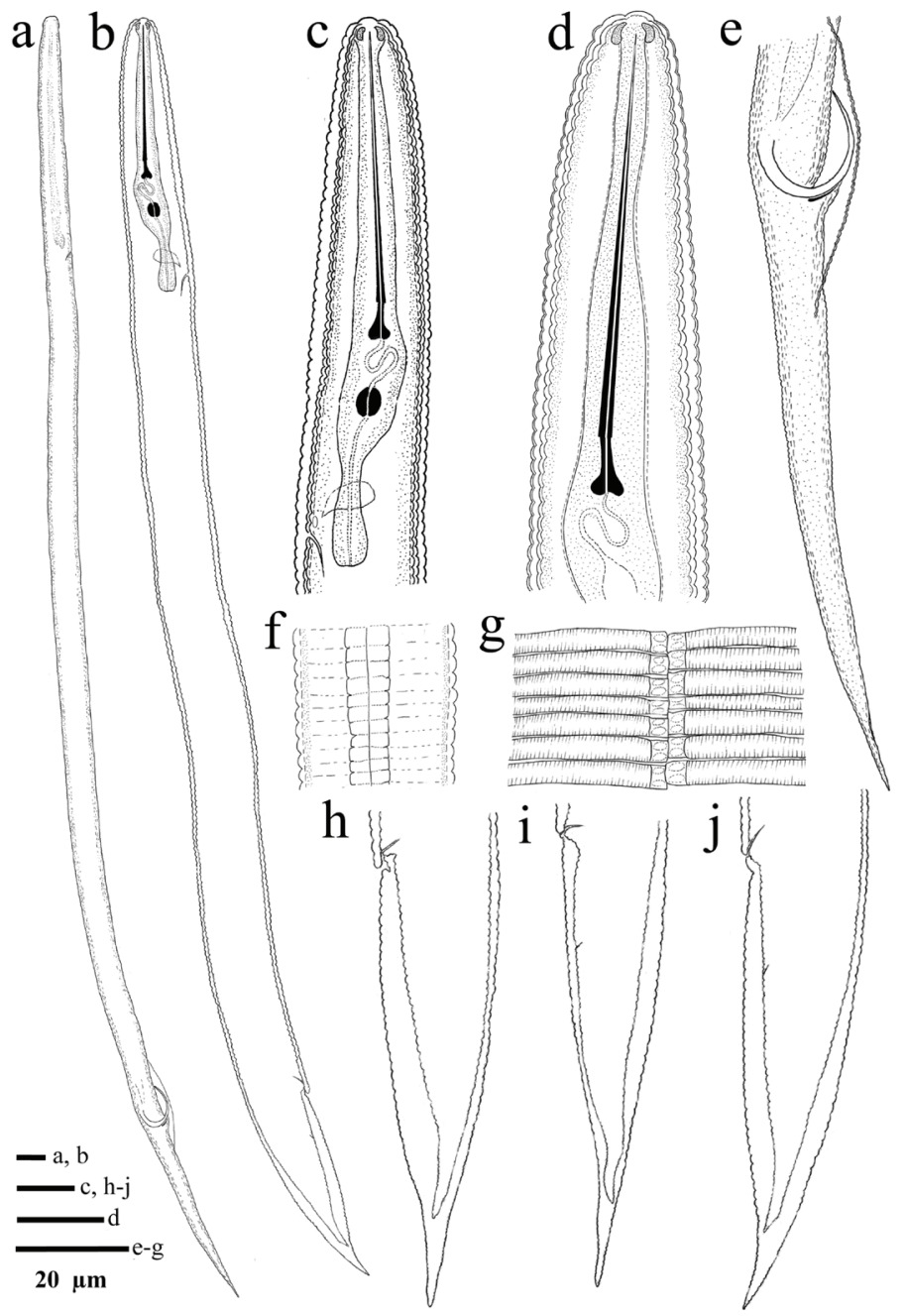

$\mathrm{a}$ - male entire body; $\mathrm{b}$ - female entire body; $\mathrm{c}$ - female pharyngeal region; $\mathrm{d}$-female anterior region; $\mathrm{e}$ - male posterior region; $\mathrm{f}, \mathrm{g}-$ - lateral lines at mid body in male and female, respectively; $\mathrm{h}-\mathrm{j}-$ female posterior region.

Figure 1. Line drawings of paratypes of Hemicycliophora paraconida $\mathrm{n}$. sp. 


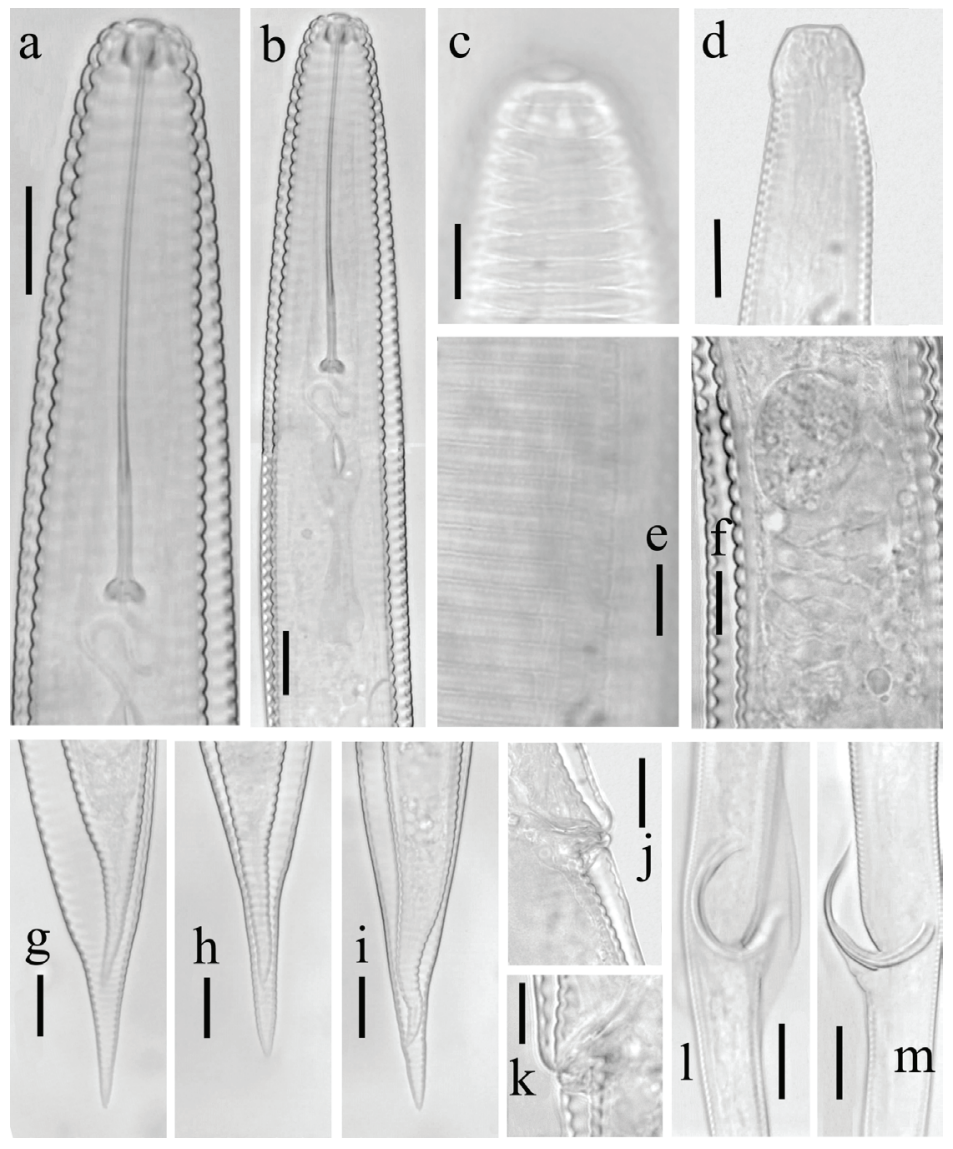

$\mathrm{a}, \mathrm{b}$ - female anterior region; $\mathrm{c}$ - surface view of male anterior region; $\mathrm{d}$ - male anterior region; e - details of lateral field in female; $\mathrm{f}$ - genital tract showing spermatheca and crustaformeria; $\mathrm{g}-\mathrm{i}$ - female tail region; $\mathrm{j}, \mathrm{k}$ - vulval region; $1, \mathrm{~m}$ - cloacal region. Scale bars: $\mathrm{a}, \mathrm{b}, \mathrm{g}-\mathrm{i}, 1, \mathrm{~m}=20 \mu \mathrm{m}, \mathrm{c}-\mathrm{f}, \mathrm{j}, \mathrm{k}=10 \mu \mathrm{m}$.

Figure 2. Photomicrographs of paratypes of Hemicycliophora paraconida $\mathrm{n}$. sp.

Description. Female. Relatively large sized nematodes (Figures 1 and 2). Body straight or irregularly arcuate upon fixation, cuticular sheath fitting closely or loosely to body, more loosely on tail and vulval region. Cuticle with coarse annulation, annuli. Annuli outside lateral field marked by a row of short scratches at their anterior and posterior border. Lateral field bordered by two distinct longitudinal lines; within the lateral field the body annuli may continue as complete transverse striae or show breaks appearing as a third longitudinal line in the middle and resulting in two longitudinal rows of blocks, each block ornamented by two vague subcuticular ovate markings, hardly visible just in few specimens (Figure 2e). Labial region broad, rounded and anteriorly truncate, continuous with body contour, but slightly narrower than body at base, bearing two distinct annuli (three in two specimens) with elevated labial disc. Stylet slender, straight or slightly curved, conus extremely larger than shaft i.e. $75-84 \%$ of total stylet length. Stylet 
knobs large and oblong to rectangular shape, posteriorly sloping creating large "cavity". Pharynx typically criconematoid with a fused procorpus and metacorpus with a large valve, a short isthmus connected to a reduced basal bulb. Secretory- Excretory pore varying in position from three annuli anterior to the base of pharynx to nine annuli posterior to it, but usually located opposite to base of basal bulb; hemizonid indistinct. Genital system mono-prodelphic occupying $26-42 \%$ of the body length, with outstretched ovary comprising oocytes in single line, spermatheca rounded to oblong in shape, offset and filled with tiny spheroid sperm cells. Crustaformeria more or less indistinct visible as a combination of small cells in few specimens; uterus thin-walled and less developed lacking a post uterus sac (PUS); vagina oblique. Vulva a wide transverse slit, not modified, vulval lips usually bearing two to three annuli appearing rounded protuberant. Tail elongate conoid with distinct annulation, tapering gradually and uniformly; approximately three times anal body diam. in length. Distal portion in some specimens slightly offset with a shallow depression at terminal one-third of tail region and tapering to a narrowly rounded to acute terminus. Intestine with indistinct lumen; rectum faintly distinguishable, most likely non-functional.

Male. Body almost cylindrical, slightly curved ventrally, marked by finer annuli than female. Labial region distinctly trapezoid, offset by distinct expansion, without annulation. Lateral fields marked by three distinct lines, two outers incisures strongly areolated. Pharynx consumptive, sometimes appearing as an open chamber. Hemizonid distinct, three to four annuli long, located three to five annuli anterior to excretory pore, marked externally by slight elevation of cuticle. Spicules robust and semi-circular; the tips slightly recurved; penial tube well-developed and almost completely covering spicule. Gubernaculum slightly curved, and slightly thickened proximally. Bursa well developed, crenate, folded over ventrally and covering spicule. Tail elongate conoid, tapering to an acute terminus.

Juvenile. Body straight or slightly ventrally arcuate. Resembling female except for lower values of body length, stylet length, and total annuli body.

Type host and locality. Specimens were recovered during July to September 2015, from the rhizosphere of undetermined grasses collected in Tilekenar Forest (coordinates: $36^{\circ} 37^{\prime} 04.9^{\prime}$ 'N 51 18 '43.6”E), Motelqo city, Mazandaran province, northern Iran.

Type material. Holotype female, five paratype females and three paratype males (Slides HPH001- HPH003) deposited in the nematode collection of the Department of Plant Protection, College of Agriculture and Natural Resources, University of Tehran, Iran. Three female paratypes and two paratype males deposited in the National Nematode Collection of the Department of Nematology, Iranian Research Institute of Plant protection, Tehran, Iran. Two paratype females and two paratype males deposited in Wageningen Nematode Collection, The Netherlands.

Polytomous key code. According to the polytomous key of Chitambar and Subbotin (2014) the new species has the following specific alphanumeric codes: A2, B7, C4, D1, E2, F1, G2, H1, I2, J2, K4, L3, M2, N1, O23, P1, Q2, R2, S3, T12, U2, V-, W2, X2, Y1

Diagnosis. Hemicycliophora paraconida $\mathrm{n}$. sp. is characterized by female being $907-1,198 \mu \mathrm{m}$ long with 286-341 body annuli. Broad labial region, anteriorly truncate, bearing two distinct annuli and an elevated labial disc. Stylet 96-115 $\mu \mathrm{m}$ long with three rounded, posteriorly sloped knobs creating a large "cavity". Lateral fields with by three longitudinal lines forming two rows of blocks with breaks and anastomosis of transverse striae; each annulus in lateral field with four hardly visible subcuticular ovate markings. Vulva not modified and vulval lips appearing rounded protuberant, bearing two to three annuli. Tail elongated uniformly conoid except for slightly marked narrower terminal third, with distinct annulation and narrowly rounded to acute 
tail end. Male with robust and semi-circular spicules with slightly recurved tips, anteriorly inside of well-developed penial tube of $15-19 \mu \mathrm{m}$.

Relationships. After molecular comparisons, four species namely $H$. conida, $H$. hellenica Vovlas, 2000, H. poranga (syn. H. ripa Van den Berg, 1981) (van den Berg et al., 2018) and $H$. halophila Yeates, 1967 shows closest similarity to the new species. Furthermore, three other species, i.e. H. iwia Brzeski, 1974, H. ovata Colbran, 1962, H. ornamenta Bajaj, 1998 are selected because of the similarity in morphological characters such as Lateral field structure, cephalic region shape, body length and female tail shape.

According to morphological, morphometrical and molecular comparisons, Hemicycliophora conida is the closest species to the new species. Table 2 represents differential morphometric characters of $H$. paraconida n. sp. and $H$. conida; the new species has a longer body length, longer stylet, more body annuli, longer tail, more posterior vulva and greater spicule compared to $H$. conida. The new species differs from $H$. iwia by having different type of lateral field (three faint lines $v s$ without lateral line often with irregular longitudinal striae), anteriorly position of vulva $(\mathrm{V}=82-84$ vs 86-94), more body annuli $(\mathrm{R}=286-341$ vs 188-219), longer tail in female and male $(\mathrm{c}=6.3-8.3$ vs $13-16.6$ and 5.5-6 vs 10.5, respectively) and sharper ending tail in both sexes. It differs from $H$. ovata, in having a slightly different lateral field (four subcuticular ovate markings $v s$ two rows of ovate ornamentations), different vulval lips (vulval lips protuberant bearing two to three annuli vs not protuberant) and different tail shape (elongated uniformly conoid $v s$ convexconoid). The new species differs from $H$. hellenica by having shorter body length (907-1197 vs 1078-1634 $\mu \mathrm{m}$ and 944-994 vs 1013-1302 $\mu \mathrm{m}$ in female and male respectively), different cephalic region (anteriorly truncate labial region with slightly elevated labial disc vs greatly protruding

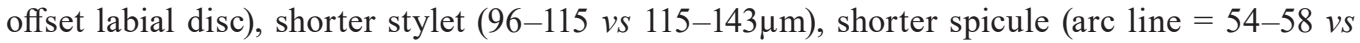
58-72 $\mu \mathrm{m}$ in arc line). It's distinguishable from $H$. poranga by greater length of female tail (c index $=6.3-8.3$ vs 8.9-12) and slightly greater stylet length (96-115 vs 82-100 $\mu \mathrm{m})$ (Chaves, 1983; Costa Manso, 1996; Crozzoli, Lamberti, 2006; Monteiro, Lordello, 1978). It differs from H. ornamenta by having slightly posteriorly position of vulva ( $\mathrm{V}=82-84 \mathrm{vs} 78-81 \%)$, less annuli number of whole body $(\mathrm{R}=286-341$ vs 355-390), different tail terminus (narrowly rounded to acute $v s$ filiform), and completely different form of cuticular ornamentation (four subcuticular ovate markings $v s$ appearing as blocks inside annuli). It differs from $H$. halophila by having more annuli number of whole body $(\mathrm{R}=286-341$ vs 190-238), greater length of female and male tail $(\mathrm{c}=6.3-8.3$ vs 9.1-14.6 and mean $=5.6$ vs 8.6 respectively), shorter spicule (arc line mean $=55.5$ $v s 68 \mu \mathrm{m}$ in arc line) and penial tube $(17 v s 22 \mu \mathrm{m})$ and shape of tail terminus (narrowly rounded to acute terminus vs rounded) (Reay, 1984; van den Berg, 1977, 1987; Yeates, 1967).

Molecular characterisation. Amplification of the D2-D3 expansion segment of 28S, sequence from Hemicycliophora paraconida $\mathrm{n}$. sp. specimens yielded a single fragment of $698 \mathrm{bp}$. The 698-bp 28S D2-D3 sequence data was less than 97.9\% homologous from any available DNA sequences from GenBank. For molecular analysis, the species with the highest identity matches in BlastN search were included. A majority consensus phylogenetic tree generated using both Bayesian and ML method of the D2-D3 of the 28S rRNA gene sequence alignment under the GTR $+\mathrm{I}+\mathrm{G}$ model is presented in Figure 3. The dataset for phylogenetic tree composed of respectively 661 total characters from which 311 characters were variable after aligning with MAFFT and manually editing. Based on the D2-D3 28S gene sequence, $H$. paraconida $\mathrm{n}$. sp. clustered with the phylogenetically most similar species H. conida (FN433875, KF430448 and KF430447) with maximal BPP and high ML BS values (0.1/99) (Fig. 3). This clade is a part of a greater clade with H. hellenica, H. onubensis van den Berg, Tiedt, Liebanas, Chitamber, Stanley, Inserra, Castillo, 
New sheath nematode, Hemicycliophora paraconida n. sp. (Rhabditida: Hemicycliophoridae)...

Table 2. Comparison of morphometrics between Hemicycliophora paraconida $\mathrm{n}$. sp. and H. conida, reported by different authors

\begin{tabular}{|c|c|c|c|c|c|c|c|c|}
\hline \multirow[b]{4}{*}{ Female (n) } & \multirow{4}{*}{$\begin{array}{c}\begin{array}{c}H . \\
\text { paraconida } \\
\text { n. sp. }\end{array} \\
\text { Iran } \\
\begin{array}{c}\text { Present } \\
\text { syudy }\end{array} \\
12\end{array}$} & \multicolumn{7}{|c|}{ H. conida Thorne, 1955} \\
\hline & & \multicolumn{2}{|c|}{ Ireland } & \multirow{2}{*}{\multicolumn{2}{|c|}{$\begin{array}{l}\text { The Netherlands } \\
\text { Loof (1968) }\end{array}$}} & \multicolumn{2}{|c|}{ Spain } & \multirow{3}{*}{\begin{tabular}{|c|} 
USA \\
$\begin{array}{c}\text { Subbotin } \\
\text { et al. } \\
(2014)\end{array}$ \\
3
\end{tabular}} \\
\hline & & \multirow{2}{*}{\begin{tabular}{|c|}
$\begin{array}{c}\text { Paratypes } \\
\text { Loof } \\
(1968)\end{array}$ \\
7
\end{tabular}} & \multirow{2}{*}{\begin{tabular}{|c|}
$\begin{array}{c}\text { Paratypes } \\
\text { Brezeski } \\
(1974)\end{array}$ \\
4
\end{tabular}} & & & \multirow{2}{*}{$\begin{array}{c}\begin{array}{c}\text { Castillo } \\
\text { et al. } \\
(1989)\end{array} \\
9\end{array}$} & \multirow{2}{*}{\begin{tabular}{|c|}
$\begin{array}{c}\text { Subbotin } \\
\text { et al. } \\
(2014)\end{array}$ \\
10
\end{tabular}} & \\
\hline & & & & 100 & 83 & & & \\
\hline $\mathrm{L}$ & $\begin{array}{c}1,017 \\
(907-1197)\end{array}$ & $700-780$ & $\begin{array}{c}810 \\
(750-850)\end{array}$ & $\begin{array}{c}840 \\
(660-990)\end{array}$ & $\begin{array}{c}790 \\
(640-870)\end{array}$ & $\begin{array}{c}817 \\
(683-912)\end{array}$ & $\begin{array}{c}770 \\
(683-912)\end{array}$ & $\begin{array}{c}836 \\
(810-873)\end{array}$ \\
\hline $\mathrm{a}$ & $\begin{array}{c}21 \\
(19.5-22.5)\end{array}$ & $18-23$ & $\begin{array}{c}24 \\
(23-26) \\
\end{array}$ & $\begin{array}{c}23 \\
(18-27) \\
\end{array}$ & $\begin{array}{c}23 \\
(18-28) \\
\end{array}$ & $\begin{array}{c}21 \\
(20-22.5)\end{array}$ & $\begin{array}{c}28.6 \\
(26-31) \\
\end{array}$ & $\begin{array}{c}25 \\
(24-26.4) \\
\end{array}$ \\
\hline $\mathrm{b}$ & $\begin{array}{c}5.3 \\
(4.6-6.1) \\
\end{array}$ & $4.9-5.2$ & $\begin{array}{c}5.5 \\
(5.3-5.7) \\
\end{array}$ & $\begin{array}{c}5.2 \\
(4.5-6) \\
\end{array}$ & $\begin{array}{c}5.1 \\
(4.1-5.7) \\
\end{array}$ & $\begin{array}{c}5.7 \\
(5.1-6.2) \\
\end{array}$ & $\begin{array}{c}5.4 \\
(5.2-5.7) \\
\end{array}$ & $\begin{array}{c}5 \\
(4.8-5.3) \\
\end{array}$ \\
\hline $\mathrm{c}$ & $\begin{array}{c}7.4 \\
(6.3-8.3)\end{array}$ & $9.5-11.4$ & $\begin{array}{c}10 \\
(9.2-10.8)\end{array}$ & $\begin{array}{c}11.1 \\
(8.7-14.4)\end{array}$ & $\begin{array}{c}11 \\
(8.3-13.5)\end{array}$ & $\begin{array}{c}10.3 \\
(9-13.7)\end{array}$ & $\begin{array}{c}11.4 \\
(10.2-12.6)\end{array}$ & $\begin{array}{c}10.1 \\
(9.6-10.4)\end{array}$ \\
\hline$c^{\prime}$ & $\begin{array}{c}3.3 \\
(2.8-3.9)\end{array}$ & - & - & - & - & $\begin{array}{c}2.7 \\
(1.9-3.2)\end{array}$ & $\begin{array}{c}2.9 \\
(1.3-3.6)\end{array}$ & $\begin{array}{c}3.5 \\
(3.5-3.6)\end{array}$ \\
\hline V & $\begin{array}{c}83.0 \\
(82-84)\end{array}$ & $85-88$ & $\begin{array}{c}86 \\
(85-87) \\
\end{array}$ & $\begin{array}{c}86 \\
(84-88) \\
\end{array}$ & $\begin{array}{c}87 \\
(83-90) \\
\end{array}$ & $\begin{array}{c}84 \\
(84-87)\end{array}$ & $\begin{array}{c}85.6 \\
(84-870\end{array}$ & $\begin{array}{c}87.3 \\
(86-88)\end{array}$ \\
\hline Stylet & $\begin{array}{c}104 \\
(96-115)\end{array}$ & $73-82$ & $\begin{array}{c}82 \\
(79-84) \\
\end{array}$ & $\begin{array}{c}87 \\
(78-96) \\
\end{array}$ & $\begin{array}{c}80 \\
(69-86) \\
\end{array}$ & $\begin{array}{c}85 \\
(77-94) \\
\end{array}$ & $\begin{array}{c}74 \\
(70-80) \\
\end{array}$ & $\begin{array}{c}83.6 \\
(77-89) \\
\end{array}$ \\
\hline Tail length & $\begin{array}{c}138 \\
(118-150)\end{array}$ & - & - & - & - & $\begin{array}{c}80 \\
(60-93)\end{array}$ & $\begin{array}{c}73 \\
(66-79) \\
\end{array}$ & $\begin{array}{c}83 \\
(78-91) \\
\end{array}$ \\
\hline $\mathrm{R}$ & $\begin{array}{c}304 \\
(286-341) \\
\end{array}$ & $180-201$ & $\begin{array}{c}202 \\
(190-211) \\
\end{array}$ & $\begin{array}{c}245 \\
(227-274) \\
\end{array}$ & $\begin{array}{c}204 \\
(180-220) \\
\end{array}$ & $\begin{array}{c}231 \\
(219-240) \\
\end{array}$ & $\begin{array}{c}238 \\
(209-278) \\
\end{array}$ & $\begin{array}{c}250 \\
(246-254)\end{array}$ \\
\hline RV (ant) & $\begin{array}{c}244 \\
(230-272)\end{array}$ & - & $\begin{array}{c}165 \\
(155-170)\end{array}$ & - & - & - & $\begin{array}{c}194 \\
(167-229)\end{array}$ & $\begin{array}{c}206 \\
(202-210)\end{array}$ \\
\hline $\mathrm{RV}$ (pos) & $\begin{array}{c}60 \\
(53-70) \\
\end{array}$ & $31-41$ & - & $\begin{array}{c}49 \\
(38-57) \\
\end{array}$ & $\begin{array}{c}41 \\
(35-46)\end{array}$ & $\begin{array}{c}46 \\
(41-51) \\
\end{array}$ & $\begin{array}{c}44 \\
(42-49) \\
\end{array}$ & \begin{tabular}{|c|}
45 \\
$(41-49)$ \\
\end{tabular} \\
\hline $\mathrm{PV} / \mathrm{ABD}$ & $\begin{array}{c}4.3 \\
(4.9-5)\end{array}$ & - & - & - & - & $\begin{array}{c}4.6 \\
(3.9-5.6)\end{array}$ & $\begin{array}{c}4.7 \\
(4-5)\end{array}$ & $\begin{array}{c}5.2 \\
(5-5.6)\end{array}$ \\
\hline Male (n) & 10 & - & - & 51 & 5 & - & - & - \\
\hline L & $\begin{array}{c}973 \\
(944-994)\end{array}$ & - & - & $\begin{array}{c}660 \\
(530-840)\end{array}$ & $620-680$ & - & - & - \\
\hline $\mathrm{a}$ & $\begin{array}{c}37 \\
(33-41)\end{array}$ & - & - & $\begin{array}{c}30 \\
(22-39)\end{array}$ & $29-33$ & - & - & \\
\hline $\mathrm{b}$ & $\begin{array}{c}7.5 \\
(6.8-8.4)\end{array}$ & - & - & $\begin{array}{c}6.1 \\
(5.1-7) \\
\end{array}$ & $5-6$ & - & - & - \\
\hline $\mathrm{c}$ & $\begin{array}{c}5.6 \\
(5.5-6) \\
\end{array}$ & - & - & $\begin{array}{c}7.2 \\
(6.4-9)\end{array}$ & $7-8$ & - & - & - \\
\hline$c^{\prime}$ & $\begin{array}{c}6.5 \\
(6-7) \\
\end{array}$ & - & - & $\begin{array}{c}5.6 \\
(4.6-6.1) \\
\end{array}$ & $4.9-5.2$ & - & - & - \\
\hline $\begin{array}{l}\text { Spicule } \\
\text { width }\end{array}$ & $\begin{array}{c}28 \\
(27-29.5)\end{array}$ & - & - & $\begin{array}{c}23 \\
(18-29)\end{array}$ & $18-20$ & - & - & - \\
\hline Tail length & $\begin{array}{c}172 \\
(166-179)\end{array}$ & - & - & $\begin{array}{c}92 \\
(79-109)\end{array}$ & $81-88$ & - & - & - \\
\hline
\end{tabular}


Subbotin 2018, H. thornei, H. poranga and H. halophila. The comparison of pairwise sequence revealed that the sequences of $28 \mathrm{~S}$ domain for the new species with other similar species were largely different and the sequence divergences are marked in the tree (Figure 3 ). The branch length of the new species clade was fairly long compared with specimens of Hemicycliophora conida, its sister species.

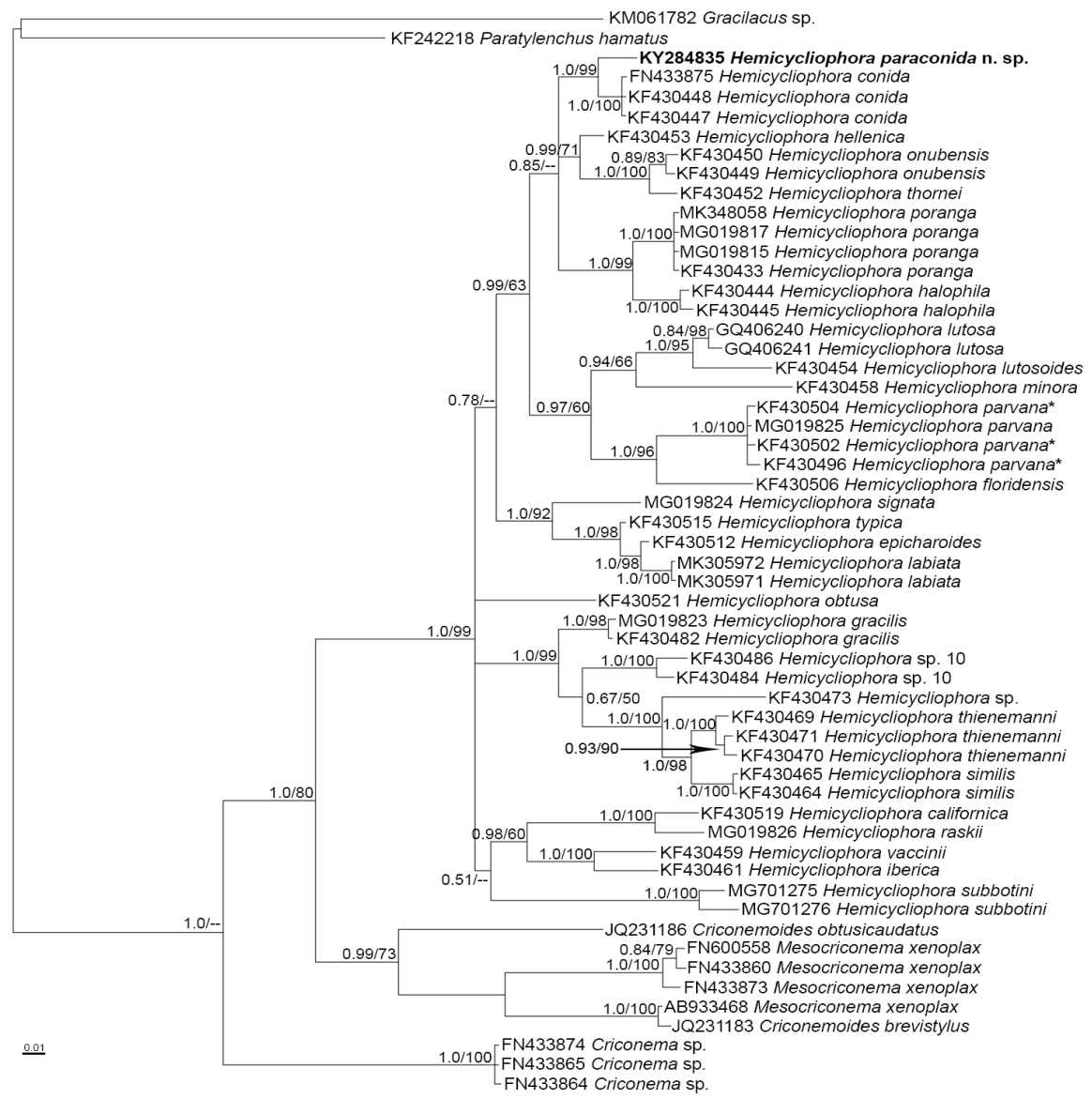

* Identified as $H$. wyei by Zeng et al. (2015).

Figure 3. Phylogenetic relationships of Hemicycliophora paraconida $\mathrm{n}$. sp. within related species and genera based on partial 28S D2/D3 under GTR $+\mathrm{I}+\mathrm{G}$ model. Bayesian posterior probabilities (BPP) and maximum likelihood bootstrap (ML/BS) values $>50 \%$ are given for appropriate clades in the form: BPP/ML BS

Alignment of the D2-D3 28S sequence obtained from the new species with the sequence from the molecularly similar species of $H$. conida isolates, yielded 692-661 total characters with 675-647 identical nucleotides (97.54-97.90\% identity). The new species forms a well-supported molecular clade with Hemicycliophora spp. (BPP/ML, 0.1/99) which is in accordance with 
Subbotin et al. (2014). However, according to the 28S tree topology, the new species clade is sister to all other Hemicycliophora as in Subbotin et al. (2014), whereas ancestral states in the D2-D3 $28 \mathrm{~S}$ gene sequence tree, it is sister to an assemblage of three paraphyletic genera, Criconema Hofmänner \& Menzel, 1914, Criconemoides Taylor, 1936 and Mesocriconema Andrássy, 1965. In our phylogram, Hemicycliophora appeared to be monophyletic, a finding that agrees with previous studies (Subbotin et al., 2014; Subbotin et al., 2005).

Because of the unevenness of deposited sequences, e.g., many species were sequenced with only one locus, either half or near-full-length of $18 \mathrm{~S}$ or $28 \mathrm{~S}$ domain and other markers, more detailed phylogenetic relationship of new species is not clear. However, both Bayesian and Maximum likelihood phylogenetic analysis suggested that the new species belongs to Clade I (Subbotin et al., 2014), which includes H. conida, H. poranga, H. halophila, H. thornei Goodey, 1963, H. hellenica, and many other undescribed/unidentified species.

Remarks. H. conida shows a wide range of morphometrical features in different populations (Brzeski, 1974; Castillo et al., 1989; Loof, 1968; Subbotin et al., 2014). The populations of this species were divided into two conspecific morphological forms only based on their morphometric features (Loof, 1968). The form I with longer female body size at least ca 230 annuli, Secretoryexcretory pore located $\mathrm{ca} 50$ annuli from the anterior end, stylet length with average of $c a 90 \mu \mathrm{m}$ and spicule chord (straight distance between extremities) $c a 23 \mu \mathrm{m}$. The form II, has 180-220 annuli in female body, excretory pore $c a 40$ annuli from the anterior end, average stylet length of $80 \mu \mathrm{m}$ and spicule width $c a 19 \mu \mathrm{m}$. The new species shows highest morphological similarity to H. conida within the genus, although molecular study proved the uniqueness of the new species. The difference between these two close species become greater when they were morphometrically compared (Table 2). However, between these two morphometrical forms of H. conida, the new species came closer to form I, although $H$. paraconida $n$. sp. was distinct from Form I with having $\mathrm{R} \geq 285$ annuli (vs. 230) in female body, $\mathrm{R}_{\mathrm{ex}}=52$ annuli (vs. 40), average stylet length of $\mathrm{ca} 104 \mu \mathrm{m}$ (vs. $90 \mu \mathrm{m}$ ) and spicule width ca $28 \mu \mathrm{m}$ (vs $23 \mu \mathrm{m})$.

\section{Discussion}

This study described a new sheath nematode species with its molecular characterization on D2-D3 expansion segment of 28S rDNA. Molecular analysis was performed based on rDNA sequence and proved this sequence region efficiency in differentiation among morphologically closed populations of Hemicycliophora.

Although Hemicycliophora spp. have many distinct morphological characters, it becomes very hard, time-consuming and somehow impossible to identify the species using only their morphology, mainly because of the overlapping in morphological features of the species and intraand inter-specific variations. It suggested that integrative taxonomy using both morphological and molecular study will increase accuracy and decrease the difficulty for identification in this species rich genus. Furthermore, approving novelty of $H$. paraconida $\mathrm{n}$. sp. as a new species using molecular study, despite having morphological similarities with $H$. conida can be considered as an example of the existence of cryptic species among the species of genus, especially those with wide range conspecific features. Furthermore the current study suggests that two conspecific forms i.e., form I and II in $H$. conida may represent two distinct species. In this regard, phylogenetic study on two conspecific forms of $H$. conida is highly recommended as small sequence divergences within the species-complexes may be interpreted as various stages in the speciation process, from recently diverged populations to distinct biological species. 
The proposal of $H$. wyei Cordero López, Robbins and Szalanski, 2013 as a junior synonym for $H$. parvana by van den Berg et al. (2018) is also approved in our molecular analysis with maximal support (BPP/ML, 0.1/100). Moreover, the results of the present study also suggest that the observed genetic diversity of Hemicycliophora is significantly higher than that shown by morphological observations. Thus, species diversity in Hemicycliophora based on morphological characters needs a thorough re-examination of the type materials of the genus. Also, our data suggest that the biodiversity of sheath nematodes is still not fully clarified and other morphological data are needed to help understanding the taxonomy and molecular phylogeny of the genus in the future.

\section{Acknowledgements}

This study was financially supported by the University of Tehran, Iran.

\section{Reference}

Andrássy, I. (1965). Verzeichnis und Bestimmungsschlussel der Arten der Nematoden Gattungen Criconemoides Taylor, 1936 und Mesocriconema n. gen. Opuscula Zoologica Budapest, 5, 153-171.

Bajaj, H.K. (1998). Criconematids of Kalesar forests and Morni hills of Haryana, India. Indian Journal of Nematology, 28, 150-158.

Brzeski, M. (1974). Taxonomy of Hemicycliophorinae (Nematoda, Tylenchida). Zeszyty Problemowe Postępów Nauk Rolniczych, 154, 237-330.

Castillo, P., Gomez-Barcina, A., Loof, P. (1989). On two species of Hemicycliophora de Man, 1921 (Nematoda: Criconematoidea) found in Spain. Nematologia mediterranea, 17.

Chaves, E. (1983). Criconematoidea (Nematoda) from Argentina. Nematologica, 29, 404-424.

Chitambar, J.J. (1993). Host range of Hemicycliophora poranga and its pathogenicity on tomato. Fundamental applied nematology, 16 (6), 557-561.

Chitambar, J.J., Subbotin, S.A. (2014). Systematics of the sheath nematodes of the superfamily Hemicycliophoroidea. Nematology Monographs and Perspectives 10 (Series Editors: Hunt, D.J. \& Perry, R.N.). Leiden, The Netherlands: Brill.

Colbran, R. (1962). Studies of plant and soil nematodes. 5. Four new species of Tylenchoidea from Queensland pineapple fields. Queensland Journal of Agricultural Science, 19, 231-239.

Cordero López, M.A., Robbins, R.T., Szalanski, A.L. (2013). Taxonomic and molecular identification of Hemicaloosia, Hemicycliophora, Gracilacus and Paratylenchus species (Nematoda: Criconematidae). Journal of Nematology, 45, 145.

Costa Manso, E. (1996). Studies on the subfamily Hemicycliophorinae (Nematoda: Criconematoidea). Fitopatologia Brasileira, 21, 197-218.

Crozzoli, R., Lamberti, F. (2006). The genus Hemicycliophora de Man, 1921 in Venezuela, with description of Hemicycliophora meridaensis sp. n. (Nematoda: Hemicycliophoridae). Russian Journal of Nematology, 14, 1-10.

De Grisse, A.T. (1969). Redescription ou modifications de quelques technique utilis [a] es dan l'etude des $\mathrm{n}$ [a] ematodes phytoparasitaires. Mededelingen van de Rijksfaculteit der Landbouwwetenschappen Gent, 34, 351-369.

de Man, J.C. (1921). Nouvelles recherches sur les nématodes libres terricoles de la Hollande. Capita Zoologica, 1, 3-62. 
Ghaderi, R., Karegar, A., Miraeiz, E., Mokaram Hesar, A. (2019). An updated and annotated checklist of the Tylenchulidae (Nematoda: Criconematoidea) of Iran. Zootaxa, 4545, 205-229.

Ghaderi, R., Kashi, L., Karegar, A. (2018). Plant-Parasitic Nematodes in Iran (based on the published reports until 2017). Iran: Agricultural Education and Extension Publication.

Goodey, T. (1963). Soil and freshwater nematodes (Second ed.). revised by J.B. Goodey, London, UK: Methuen.

Hofmaenner, B., Menzel, R. (1914). Neue arten freilebender nematoden aus der schweiz, 44.

Huson, D. H., Scornavacca, C. (2012). Dendroscope 3: an interactive tool for rooted phylogenetic trees and networks. Systematic biology, 61, 1061-1067.

Katoh, K., Standley, D.M. (2013). MAFFT multiple sequence alignment software version 7: improvements in performance and usability. Molecular biology evolution, 30, 772-780.

Larget, B., Simon, D.L. (1999). Markov chain Monte Carlo algorithms for the Bayesian analysis of phylogenetic trees. Molecular biology and evolution, 16, 750-759.

Loof, P. (1968). Taxonomy of Hemicydiophora species from west and central Europe (Nematoda: Criconematoidea). Mededelingen van de Landbouwhogeschool te Wageningen, 68, 1-43.

Loof, P. (1984). Hemicycliophora species from Iran (Nematoda: Criconematoidea). Nematologica, 30, $22-41$.

Loos, C. (1948). Notes on free-living and plant-parasitic nematodes of Ceylon 3. Ceylon Journal of Science. Section B. Zoology Geology, 23, 119-124.

Maria, M., Cai, R., Qu, N., Castillo, P., Zheng, J. (2018). Morphological and molecular characterization of Hemicycliophora subbotini $\mathrm{n}$. sp. (Tylenchida: Hemicycliophoridae) from China. Zootaxa, 4433, $161-173$.

Monteiro, A. R., Lordello, L.G.E. (1978). Description of Hemicycliophora poranga n. sp. from Brazil (Nemata). Revista brasileira de biologia, 38, 569-571.

Nunn, G. (1992). Nematode molecular evolution. In. Ph.D. Dissertation, University of Nottingham, UK.

Nylander, J. (2004). MrModeltest v2. Evolutionary Biology Centre, Uppsala University. Retrieved from: https://github.com/nylander/MrModeltest2.

Raski, D. (1958). Four new species of Hemicycliophora de Man, 1921, with further observations on H. brevis Thorne, 1955 (Nematoda: Criconematidae). Proceedings of the Helminthological Society of Washington, 25, 125-131.

Raski, D., Luc, M. (1987). A reappraisal of Tylenchina (Nemata): 10. The superfamily Criconematoidea Taylor, 1936. Revue de Nématologie, 10, 409-444.

Ray, S., Das, S. (1978). Hemicaloosia americana n. gen., n. sp. (Nematoda: Hemicycliophoridae) from Orissa, India. OUAT Journal of Research, 8, 131-138.

Reay, F. (1984). Plant nematodes from Australia: studies on Hemicycliophoridae (Nematoda: Tylenchida). Revue nematol, 7, 367-384.

Ronquist, F., Huelsenbeck, J.P. (2003). MrBayes 3: Bayesian phylogenetic inference under mixed models. Bioinformatics, 19, 1572-1574.

Siddiqi, M.R., Goodey, J.B. (1964). The status of the genera and subfamilies of the Criconematidae (Nematoda); with a comment oh the position of Fergusobia. Nematologica, 9, 363-377.

Silvestro, D., Michalak, I. (2012). raxmlGUI: a graphical front-end for RAxML. Organisms Diversity Evolution, 12, 335-337.

Skarbilovich, T. (1959). On the structure of systematics of nematodes order Tylenchida Thorne, 1949. Acta Parasitologica Polonica, 7, 117-132. 
Subbotin, S.A., Chitambar, J.J., Chizhov, V.N., Stanley, J.D., Inserra, R.N., Doucet, M.E., et al. (2014). Molecular phylogeny, diagnostics, and diversity of plant-parasitic nematodes of the genus Hemicycliophora (Nematoda: Hemicycliophoridae). Zoological Journal of the Linnean Society, 171, 475-506.

Subbotin, S.A., Vovlas, N., Crozzoli, R., Sturhan, D., Lamberti, F., Moens, M., et al. (2005). Phylogeny of Criconematina Siddiqi, 1980 (Nematoda: Tylenchida) based on morphology and D2-D3 expansion segments of the 28S-rRNA gene sequences with application of a secondary structure model. Nematology, 7, 927-944.

Tarjan, A.C. (1952). The nematode genus Hemicycliophora de Man, 1921 (Criconemnatidae) with a description of a new plant-parasitic species. Proceedings of the Helminthological Society of Washington, 19 (2), 65-77.

Taylor, A. (1936). The genera and species of the Criconematinae, a sub-family of the Anguillulinidae (Nematoda). Transactions of the American Microscopical Society, 55, 391-421.

Thorne, G. (1955). Fifteen new species of the genus Hemicycliophora with an emended description of H. cypica de Man Tylenchida Criconematidae. Proceedings of the Helminthological Society of Washington, 22, 1-16.

van den Berg, E. (1977). Descriptions of new and little known Criconematidae from South Africa (Nematoda). Phytophylactica, 9, 95-101.

van den Berg, E. (1981). Further studies on the genus Hemicycliophora de Man, 1921 in South Africa (Nematoda: Hemicycliophoroidea) with a description of a new species. Phytophylactica, 181-194.

van den Berg, E. (1987). New Nematodes from the mountains of the western Cape (Criconematina: Nematoda). Phytophylactica, 19, 399-404.

van den Berg, E., Tiedt, L.R., Liébanas, G., Chitambar, J.J., Stanley, J.D., Inserra, R.N., et al. (2018). Morphological and molecular characterisation of two new Hemicycliophora species (Tylenchida: Hemicycliophoridae) with a revision of the taxonomic status of some known species and a phylogeny of the genus. Nematology, 20, 319-354.

Vovlas, N. (2000). Hemicycliophora hellenica n. sp. (Nemata: Criconematidae) from Greece. Journal of nematology, 32, 35.

Whitehead, A., Hemming, J. (1965). A comparison of some quantitative methods of extracting small vermiform nematodes from soil. Annals of applied Biology, 55, 25-38.

Williams, B.D., Schrank, B., Huynh, C., Shownkeen, R., Waterston, R. (1992). A genetic mapping system in Caenorhabditis elegans based on polymorphic sequence-tagged sites. Genetics, 131, 609-624.

Yeates, G. (1967). Studies on nematodes from dune sands. 8. Hemicycliophora halophila n. sp. and Ereptonema inflatum n. sp. New Zealand journal of science, 10, 802-807.

Zeng, Y., Ye, W., Kerns, J., Tredway, L., Martin, S., Martin, M. (2015). Molecular characterization and phylogenetic relationships of plant-parasitic nematodes associated with turfgrasses in North Carolina and South Carolina, United States. Plant disease, 99, 982-993.

Cite as: Miraeiz, E., Ghorbanzad, H., Esmaeili, M., Heydari, R. (2020). New sheath nematode, Hemicycliophora paraconida $\mathrm{n}$. sp. (Rhabditida: Hemicycliophoridae) from Northern forest in Iran. Acta Biologica, 27, 43-56. DOI: 10.18276/ab.2020.27-05. 